\title{
The dineolignan from Saururus chinensis, manassantin B, inhibits tumor-induced angiogenesis via downregulation of matrix metalloproteinases 9 in human endothelial cells
}

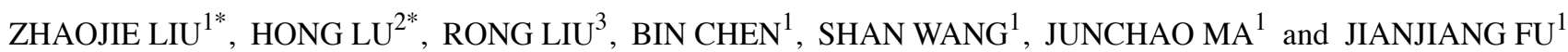 \\ ${ }^{1}$ Department of Pharmacology, College of Pharmacy and ${ }^{2}$ Network and Educational Technology Center, \\ Jiangxi University of Traditional Chinese Medicine, Nanchang, Jiangxi 330004; ${ }^{3}$ State Key Laboratory \\ of Food Science and Technology, Nanchang University, Nanchang, Jiangxi 330047, P.R. China
}

Received January 3, 2014; Accepted March 10, 2014

DOI: $10.3892 /$ or.2014.3244

\begin{abstract}
Manassantin B (MB) is a neolignan isolated from Saururus chinensis that exhibits a range of activities, including anti-inflammatory, antiseptic and antitumor activity. MB was recently found to affect cell adhesion and expression of several adhesion molecules. Based on the important roles of these adhesion molecules in angiogenesis, we evaluated a possible role for $\mathrm{MB}$ in tumor-induced angiogenesis in endothelial cells (ECs). In the present study, we found that MB blocked tumor-induced tube formation of ECs and significantly inhibited the invasion of ECs through the reconstituted basement membrane. MB suppressed the activity of matrix metalloproteinases (MMPs) and downregulated the expression of matrix metalloproteinases 9 . Western blotting showed reduction of RUNX2 activation by MB. RUNX2 transcription factor assay and chromatin immunoprecipitation assay showed that the interaction between RUNX2 and target sequences in the matrix metalloproteinases 9 promoters was inhibited by MB. Our findings suggested that the inhibitory effects of MB on tumor-induced angiogenesis were caused by matrix metalloproteinases 9 inhibition, which was associated with the downregulation of RUNX2 transcriptional activity.
\end{abstract}

\section{Introduction}

Angiogenesis, the development of novel blood vessels, is a critical event in many important disease states including ischemic cardiovascular disease, wound healing, inflammation,

Correspondence to: Dr Jianjiang Fu, Department of Pharmacology, College of Pharmacy, Jiangxi University of Traditional Chinese Medicine, Nanchang, Jiangxi 330004, P.R. China

E-mail: jianjiang.fu@gmail.com

*Contributed equally

Key words: manassantin B, angiogenesis, matrix metalloproteinases 9, RUNX2, Saururus chinensis psoriasis, primary tumor growth and formation of metastases. It is a highly complex process involving endothelial cell (EC) activation, disruption of vascular basement membranes, and migration and proliferation of ECs (1). This process is orchestrated by a large number of cytokines and associated receptors and proteinases, such as vascular endothelial growth factor (VEGF), fibroblast growth factor, interleukin 8 and matrix metalloproteinases (MMPs) (2). Tumor-induced angiogenesis is a process of irregular blood vessel formation, which results in structurally and functionally abnormal blood vessels. This abnormality impairs effective delivery of therapeutic agents to all regions of tumors, it creates abnormal properties of cancer cells, such as survival, resistance of radiation and chemotherapy, and selects for more malignant cells (3). Several decades ago, Dr Folkman proposed anti-angiogenic therapy as an effective method for treatment of cancer $(4,5)$. Nowadays, a number of inhibitors targeting the tumor vasculature have been identified to improve the sensitivity of cancer cells to cytotoxic chemotherapy and other therapeutics (6-8).

Saururus chinensis (S. chinensis) is a perennial herbaceous plant that grows wild in moist and shady places throughout East Asia (9). It has a long history of use in China as a folk medicine to remedy various edema, gonorrhea, jaundice and inflammatory diseases (9-11). Previous phytochemical and pharmacological studies on this plant have revealed several types of secondary metabolites, such as lignans, neolignans, aristolactams and flavonoids, as the biologically active compounds $(12,13)$. Numerous studies reported anticancer activities of lignans isolated from S. chinensis. Seo et al (14) reported significant effects of Saucernetin-7, a dineolignan from this herb, on proliferation, cell cycle regulation and differentiation of HL-60 cells. Other groups described the anticancer-related effects of these lignans, such as antiinvasive effects and anti-adhesive properties. Manassantin A and $\mathrm{B}$, dineolignan compounds isolated from $S$. chinensis roots, inhibited PMA-induced ICAM-1 expression in HL-60 cells in a dose-dependent manner (15). Overexpression of MMP-9 induced by lipopolysaccharide (LPS) was suppressed by Saucerneol G, a new lignan from $S$. chinensis, in RAW264.7 cells (16). These findings suggested that bioactive lignans from $S$. chinensis exhibit anti-adhesion, anti-migration and 
anti-invasion activities in several cell types and may contain properties to inhibit the malignant progression of tumors.

Based on these findings, we investigated whether bioactive lignans from $S$. chinensis impair EC activation and angiogenesis in the progression of solid tumors. We focused on the anti-angiogenic effects of lignans isolated from $S$. chinensis. By invasion-inhibition-guide fractionation, we found that $\mathrm{MB}$, one of the dineolignans isolated from $S$. chinensis, exhibited strong inhibitory effects on EC invasion in vitro. In the present study, we demonstrated that MB exhibited anti-angiogenic effects in ECs, and these effects may be mediated by inhibition of MMPs via downregulation of the transcriptional activity of RUNX2.

\section{Materials and methods}

Materials. HPLC grade MB (Fig. 1) was purchased from AppliChem GmbH (Cat. No. A9007; St. Louis, MO, USA). A stock solution $(1 \mathrm{mM})$ was prepared by dissolving $\mathrm{MB}$ in dimethyl sulfoxide (DMSO). Recombinant human VEGF165 (Cat. No. 293-VE) is a product of R\&D Systems (Minneapolis, MN, USA). L-sulforaphane (SFP; Cat. No. S6317; SigmaAldrich, Beijing, China), a potent inducer of inhibition of angiogenesis, and batimastat (Bat; Cat. No. SC-203833; Santa Cruz Biotechnology, Inc., Santa Cruz, CA, USA), a broad spectrum MMP inhibitor, were used in the present study and served as positive controls.

Cell culture and animals. Human umbilical vein endothelial cells (HUVECs) were isolated as previously described (17) from umbilical cords obtained from a parturient at the Department of Maternity at the First Affiliated Hospital of Nanchang University, who provided written informed consent. The study protocol was approved by the Institutional Review Board (IRB) of Nanchang University. HUVECs were routinely grown in M199 supplemented with $10 \%$ fetal bovine serum (FBS) (both from Gibco, Grand Island, NY, USA) and EC growth supplement (BD Biosciences, Bedford, MA, USA) at $37^{\circ} \mathrm{C}$ and $5 \% \mathrm{CO}_{2}$. HUVECs between P3 and P4 were used for all experiments. The human umbilical vein cell line, EA.hy 926, was purchased from the cell bank of Shanghai Institute for Biological Sciences, CAS. EA.hy 926 cells were maintained in Dulbecco's modified Eagle's medium supplemented with $10 \% \mathrm{FBS}, 100 \mathrm{IU} / \mathrm{ml}$ penicillin and $100 \mu \mathrm{g} / \mathrm{ml}$ streptomycin (Invitrogen, Beijing, China) in a humidified incubator containing $5 \% \mathrm{CO}_{2}$ at $37^{\circ} \mathrm{C}$. Prior to performing each assay, the ECs were serum starved for $4 \mathrm{~h}$.

Five to six-week-old male Sprague Dawley (SD) rats were purchased from the Vital River Laboratories (Beijing, China) to obtain thoracic aorta in the rat aortic ring angiogenesis assay. All animal experiments were conducted in strict accordance with full ethical approval of the Animal Ethics Committees of Jiangxi University of Traditional Chinese Medicines. All surgery was performed under sodium pentobarbital anesthesia, and all efforts were made to minimize suffering.

Tube formation assay. The tube formation assay was used to investigate the effects of MB on angiogenesis in vitro. Briefly, $80 \mu 1$ of liquid growth factor-reduced Matrigel (BD Biosciences, San Jose, CA, USA) were added to each well of a 96-well plate.

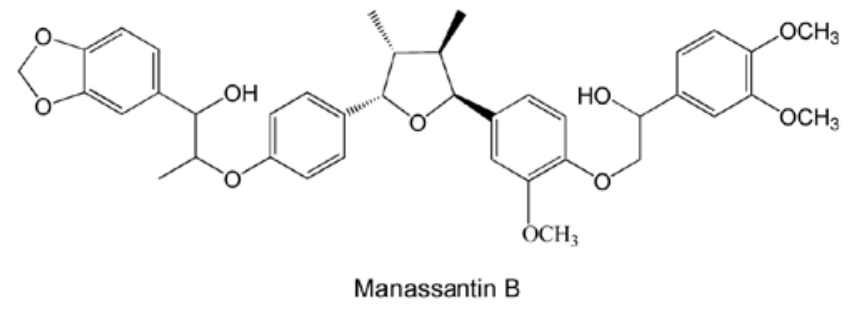

Figure 1. Molecular structure of manassantin B.

After $45 \mathrm{~min}$ incubation at $37^{\circ} \mathrm{C}, 3 \times 10^{4} \mathrm{ECs} /$ well in $100 \mu 1$ complete culture medium containing vehicle, MB or SFP was seeded in each well. Then, $100 \mu \mathrm{l}$ serum-free medium containing VEGF (final concentration $2 \mathrm{ng} / \mathrm{ml}$ ) was added. After $24 \mathrm{~h}$ incubation at $37^{\circ} \mathrm{C}$ and $5 \% \mathrm{CO}_{2}$, the images of each well were recorded by an inverted microscope (Leica DMI3000B, Germany).

Cell proliferation assay. Cell proliferation was assayed by 3-(4,5-dimethylthiazol-2-yl)-2,5-diphenyltetrazolium bromide (MTT) assay. Briefly, log-phase cells were seeded in 96-well plates $24 \mathrm{~h}$ before initiation of treatment with MB in the presence of $2 \mathrm{ng} / \mathrm{ml}$ VEGF. The vehicle-treated cells served as controls. Three duplicate wells were set up in each sample. After incubation with a range of MB or vehicle for $24 \mathrm{~h}$, cells were incubated with MTT (final concentration $0.5 \mathrm{mg} / \mathrm{ml}$ ) for $4 \mathrm{~h}$ at $37^{\circ} \mathrm{C}$ (Sigma-Aldrich). The media was carefully removed from each well and $150 \mu \mathrm{l}$ of DMSO was added. The plates were gently agitated and optical absorbance at OD $570 \mathrm{~nm}$ and OD $450 \mathrm{~nm}$ was determined using a microplate reader (ELx800; BioTek, Winooski, VT, USA). Vehicle only-treated cells served as the indicator of $100 \%$ cell viability. Each experiment was repeated three times.

Rat aortic ring angiogenesis assay. In the present study, an $e x$ vivo tube formation system was used as previously described (18) to evaluate the anti-angiogenic effect of MB. Male SD rats (5-6 weeks old) were euthanized and thoracic aortas were retrieved. After rinsing with $1 \%$ antibiotic/antimycotic cocktail in 1X PBS (100 U/ml penicillin, $100 \mu \mathrm{g} / \mathrm{ml}$ streptomycin and $0.25 \mu \mathrm{g} / \mathrm{ml}$ amphotericin B; Invitrogen), the surrounding fibroadipose tissue of thoracic aorta was completely removed with fine microdissection scissors, and the thoracic aorta was cut into $1 \mathrm{~mm}$ rings with a scalpel blade. Then, all the rings were implanted on a Matrigel-coated 96-well microtiter plate. Matrigel was added again to embed and fix rings. After $30 \mathrm{~min}$ incubation in $5 \% \mathrm{CO}_{2}$ at $37^{\circ} \mathrm{C}$, the aorta rings were incubated in human endothelial serumfree medium (Gibco, Carlsbad, CA, USA) supplemented with $2 \% \mathrm{FBS}, 50 \mathrm{U} / \mathrm{ml}$ penicillin and $50 \mu \mathrm{g} / \mathrm{ml}$ streptomycin (Invitrogen) for $24 \mathrm{~h}$ and then treated with different doses of MB for 13 days. Finally, $8 \mu \mathrm{g} / \mathrm{ml}$ Calcein AM (BD Biosciences) was added to stain microvessels. Images of the microvessels were obtained using an inverted fluorescence microscope (Leica DMI3000B).

In vitro assay of EC invasion. Effects of $\mathrm{MB}$ on invasion of ECs were measured by a 48 -well microchemotaxis system (AP48; Neuro Probe, Gaithersburg, MD, USA). Briefly, $5 \mu \mathrm{g}$ 
of fibronectin in a volume of $50 \mu \mathrm{l}$ was applied on the rough (lower) surface of the polycarbonate membrane, and $5 \mu \mathrm{g} /$ filter Matrigel was plated to the smooth (upper) surface. The lower chambers of the plates were then filled with $30 \mu \mathrm{l}$ medium containing $0.1 \%$ BSA. Log-phase cells were harvested and re-suspended in culture medium with $0.1 \%$ BSA. Cell suspensions (100 $\mu \mathrm{l}$ containing $1 \times 10^{5}$ cells) and $2 \mathrm{ng} / \mathrm{ml}$ VEGF were added to the upper compartment and incubated for $16 \mathrm{~h}$ at $37^{\circ} \mathrm{C}$ in a $5 \% \mathrm{CO}_{2}$ atmosphere. After incubation, the filters were fixed with methanol and stained with $0.5 \%$ crystal violet for $60 \mathrm{~min}$. The cells on the upper surface of the filters were removed by wiping with cotton swabs. The cells invading to the lower surface of the filter through Matrigel and filter were quantified with the Image-Pro Plus software 5.0 (Media Cybernetics Inc., Silver Spring, MD, USA) and the most representative results are illustrated in the figures. Each assay was performed in triplicate.

Zymography analysis. MMP activity was analyzed with gelatin zymograms. Following $24 \mathrm{~h}$ treatment with MB, Bat or vehicle in the presence of $2 \mathrm{ng} / \mathrm{ml} \mathrm{VEGF,} \mathrm{ECs} \mathrm{were} \mathrm{harvested} \mathrm{and}$ lysed. Cell lysates were diluted in $50 \mathrm{mM}$ Tris- $\mathrm{HCl}, \mathrm{pH} 7.4$, and separated by electrophoresis in $7.5 \%$ sodium dodecyl sulfate polyacrylamide gels containing $2 \mathrm{mg} / \mathrm{ml}$ gelatin. After electrophoresis, the gels were washed in $2.5 \%$ Triton $\mathrm{X}-100$ for $30 \mathrm{~min}$ and then incubated for $16 \mathrm{~h}$ at $37^{\circ} \mathrm{C}$ in $50 \mathrm{mM}$ Tris- $\mathrm{HCl}$, $\mathrm{pH} 7.4,200 \mathrm{mM} \mathrm{NaCl}, 10 \mathrm{mM} \mathrm{CaCl} \mathrm{Cl}_{2}$. Gels were then stained with Coomassie brilliant blue R-250 and destained with $40 \%$ methanol-10\% acetic acid until clear bands appeared. Images were captured with the UVP EC3 gel imaging system.

Fluorescence resonance energy transfer-based MMP activity assay. MMP activities were also measured by the SensoLyte ${ }^{\circledR}$ 570 Generic MMP Assay kit (AnaSpec, Fremont, CA, USA). This kit uses a fluorescence resonance energy transfer (FRET)based method to detect the activities of a variety of MMPs including MMP-1, -2, -3, -7, -8, -9, -10, -11, -12, -13 and -14. It uses 5-FAM (fluorophore) and QXL520 ${ }^{\mathrm{TM}}$ (quencher) labeled FRET peptide substrates for continuous measurement of MMP activities. In an intact FRET peptide, the fluorescence of 5 -FAM is quenched by SensoLyte ${ }^{\circledR}$. Upon cleavage of FRET peptide by MMPs, the fluorescence of 5-FAM is recovered. Analyses were performed according to the manufacturer's instructions. Briefly, supernatants of ECs were collected after incubation with or without MB for $24 \mathrm{~h}$. MMPs in supernatants were activated by incubation with 4-aminophenylmercuric acetate for $1 \mathrm{~h}$ at $37^{\circ} \mathrm{C}$. Fifty microliter MMP-containing samples and $50 \mu \mathrm{l}$ MMP substrate solution were added into a 96-well plate. The reagents were mixed by shaking the plate gently for $30 \mathrm{sec}$. After a $50-\mathrm{min}$ incubation period at $37^{\circ} \mathrm{C}$, the reaction was stopped by adding stop solution, and fluorescence intensity was measured by a multilabel counter (Victor $3^{\mathrm{TM}}$; Perkin-Elmer, Waltham, MA, USA) at Ex/Em = 540/575 nm.

Western blot analysis. Cells were rinsed twice with PBS, and total proteins were extracted in $500 \mu \mathrm{l}$ lysis buffer. Aliquots of whole cell lysates were separated by $10 \%$ SDS-PAGE and then transferred to Hybond nitro blotting membranes. The membranes were blocked with 3\% BSA in Tris-buffered saline containing $0.5 \mathrm{ml} / 1$ Tween-20 and then incubated with primary antibodies against MMP-9 (SC-6840), RUNX2 (SC-10758) (both from Santa Cruz), and phospho-RUNX2 (AP3559a; Abgent), followed by incubation with horseradish peroxidase (HRP)-conjugated secondary antibodies. Immunoreactive proteins were detected using an enhanced chemiluminescence kit (Millipore). $\beta$-actin (SC-130301; Santa Cruz) served as an internal control.

RUNX2 transcription factor assay. RUNX2 activity in EC nuclear extracts was detected using the TransAM ${ }^{\mathrm{TM}}$ AML-3/RUNX2 kit (Active Motif North America, Carlsbad, CA, USA) following the manufacturer's instructions. Cell extracts were prepared using the Nuclear Extract kit (Active Motif) with ECs that were treated with either MB or DMSO for $24 \mathrm{~h}$. Then, $20 \mu \mathrm{l}$ of extracts diluted in complete lysis buffer and containing $15 \mu \mathrm{g}$ nuclear extract were added into a 96-well plate. This plate immobilizes oligonucleotides containing RUNX2 consensus binding sites. Saos-2 nuclear extract served as a positive control for RUNX2 activation, and $20 \mu \mathrm{l}$ complete lysis buffer served as the blank. The wild-type consensus oligonucleotide was provided as a competitor for RUNX2 binding to monitor the specificity of the assay. After $1 \mathrm{~h}$ incubation at room temperature, the plate was washed three times with washing buffer. Diluted primary antibody (100 $\mu \mathrm{l})$ was added into wells and incubated for $1 \mathrm{~h}$ at room temperature without agitation. After three washes, HRP-labeled secondary antibody was added and incubated for $1 \mathrm{~h}$ at room temperature. Then, $100 \mu \mathrm{l}$ developing solution was added to initiate the color reaction. After $100 \mu \mathrm{l}$ stop solution was added, the absorbance was measured within $5 \mathrm{~min}$ at $450 \mathrm{~nm}$ with a reference wavelength of $655 \mathrm{~nm}$ using an ELx800 microplate reader (BioTek).

Chromatin immunoprecipitation assay. Chromatin immunoprecipitation assay (ChIP) was performed using the agarose ChIP kit as described in the manufacturer's instructions (Pierce $^{\mathrm{TM}}$ Agarose ChIP kit; Thermo Scientific). Briefly, crosslinking was performed for 10 min using formaldehyde (final concentration 1\%). Crosslinked cells then were lysed with lysis buffer containing protease inhibitors. To obtain DNA fragments with an average size of $0.3 \mathrm{~kb}$, micrococcal nuclease was added and samples were incubated for $5 \mathrm{~min}$. Protein-DNA complexes were immunoprecipitated using anti-RUNX2 antibody (SC-10758; Santa Cruz). Normal rabbit IgG served as a negative control and anti-RNA polymerase II antibody served as positive control. After DNA recovery, purified DNA was subjected to PCR amplification on the Mastercycler gradient (Eppendorf) using the Phusion ${ }^{\circledR}$ HighFidelity PCR kit. The primers designed to amplify one of the RUNX2 binding regions (-220 bp; TGGGGTC) in the human mmp-9 promoter (GeneBank no. NM_004994) were: forward, 5'-ACA GTT CCC ACA AGC TCT GC-3' and reverse, 5'-CAG CAT GAG AAA GGG CTT ACA-3'. The PCR profile was: $15 \mathrm{~min}$ at $95^{\circ} \mathrm{C}$, followed by 30 three step cycles of $15 \mathrm{sec}$ at $95^{\circ} \mathrm{C}, 30 \mathrm{sec}$ at $58^{\circ} \mathrm{C}$ and $30 \mathrm{sec}$ at $72^{\circ} \mathrm{C}$. PCR reactions were subjected to a final extension at $72^{\circ} \mathrm{C}$ for $10 \mathrm{~min}$. PCR analysis was performed using the Mastercycler gradient. Aliquots of total DNA before immunoprecipitation were saved as input, and these input lysates were also processed as above. All ChIP assays were performed at least three times, and the most representative results are illustrated in the figures. 
A
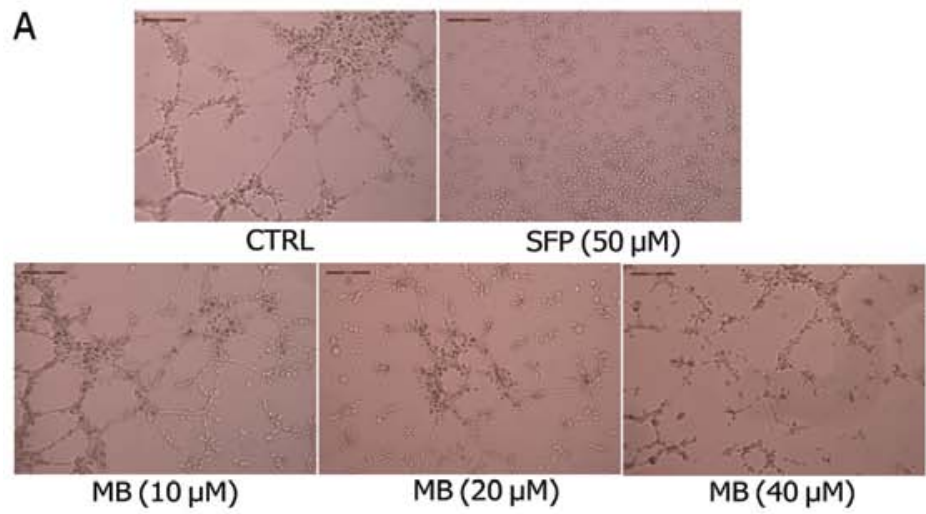

$\mathrm{MB}(10 \mu \mathrm{M})$

B

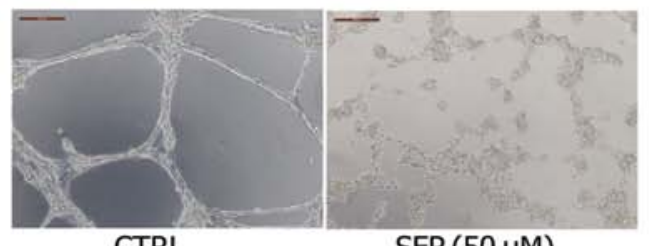

CTRL

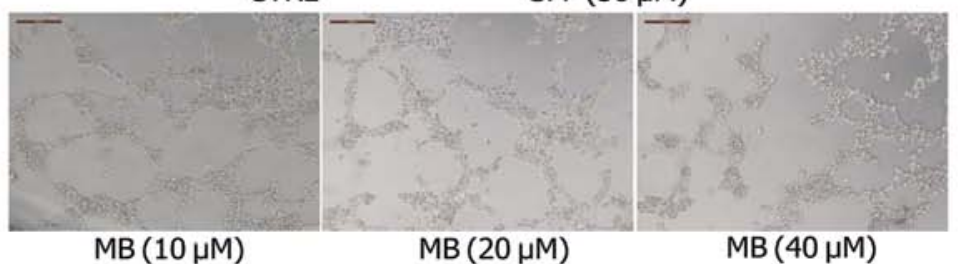

C

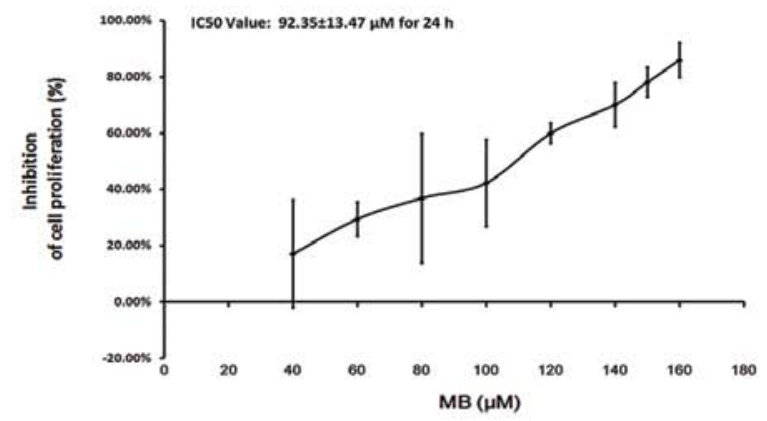

D

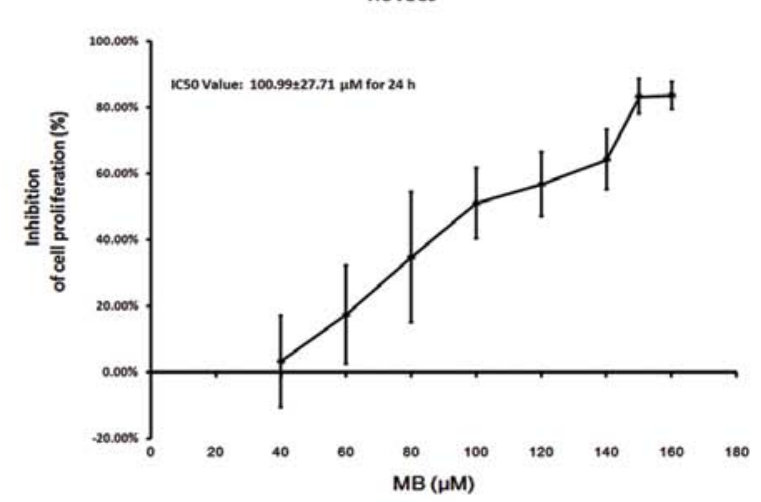

Figure 2. In vitro tube formation of ECs is significantly affected by MB in a dose-dependent manner. (A) MB inhibited mesh-like structure formation of EA.hy 926 cells on Matrigel. (B) Effects of MB on tube formation of HUVECs. In vitro tube formation assay was carried out as described in Materials and methods. MB showed a slight suppression on (C) EA.hy 926 and (D) HUVEC growth. Cell proliferation was evaluated by MTT assay. Values were normalized to the mean number of adherent control cells and presented as means \pm SD percentage $(n=3)$. Vehicle (DMSO) was used as a CTRL, and SFP served as the positive CTRL. Each assay was performed in triplicate. ECs, endothelial cells; MB, manassantin B; HUVECs, human umbilical vein endothelial cells; SFP, L-sulforaphane.

Statistical analysis. The data are presented as means $\pm \mathrm{SD}$ and were analyzed with SPSS for Windows (13.0) software program (Chicago, IL, USA). Comparison among different groups was carried out by one-way analysis of variance (the one-way ANOVA). Differences between means were considered statistically significant at $\mathrm{P}<0.05$.

\section{Results}

Effects of $M B$ on tube formation of ECs in vitro and ex vivo. We first used an in vitro tube formation assay to assess the effects of MB on tumor-induced angiogenesis. As shown in Fig. 2A, when EA.hy 926 cells were seeded on Matrigel, capillary-like structures with a lumen were formed. After exposure to MB solution at various concentrations for $24 \mathrm{~h}$, these structures were destroyed in a dose-dependent manner. MB treatment of HUVECs, a primary EC line derived from the endothelium of umbilical vein, showed that normal capillary-like structures were also significantly impaired by MB (Fig. 2B). Fig. 2C and D shows the growth inhibition effects of MB on EA.hy 926 and HUVECs; the $\mathrm{IC}_{50}$ values of MB were $92.35 \pm 13.47$ and $100.99 \pm 27.71 \mu \mathrm{M}$ and the inhibitory rates of $40 \mu \mathrm{M} \mathrm{MB}$ were 17.06 and $3.24 \%$, respectively. These data indicate that the observed ability of MB to suppress tube formation was not due to growth inhibition.

Next, the rat aortic ring angiogenesis assay was employed to verify the anti-angiogenic effects of MB. New blood vessels, triggered by the injury of the dissection procedure and mediated by growth factors produced from the aortic ring, were demolished by MB in a dose-dependent manner (Fig. 3).

Effects of $M B$ on tumor-induced invasion of ECs in vitro. Invasion of ECs through the basement membrane to form sprouting vessels is essential to angiogenesis. Thus, we next examined the anti-invasive effects of MB on ECs. As shown in Fig. 4A, MB significantly blocked the transmembrane invasion of EA.hy 926 cells. The invasion rate across the reconstituted basement membrane was $79.43 \%, 64.60 \%$ and $38.49 \%$ when the cells were incubated with 10,20 and $40 \mu \mathrm{M}$ MB for $14 \mathrm{~h}$, respectively, compared with controls. Similar results were obtained with HUVECs (Fig. 4B). Collectively, these results demonstrated that MB significantly inhibited the invasion through the basement membrane of ECs, and this effect may contribute to the anti-angiogenic properties of MB.

Effects of $M B$ on MMP-9 activity and expression in ECs. Breakdown of the extracellular matrix by MMPs in surrounding tissues is a fundamental step of EC invasion in tumor-induced angiogenesis. Therefore, we examined the effects of MB on MMPs in ECs. Fig. 5 shows the effects of MB on MMPs in EA.hy 926 and HUVECs. Data from the zymography analysis showed that MB significantly inhibited hydrolyzation of gelatin in both EC lines (Fig. 5A). Using a FRET-based analysis, we found that MB exhibited signifi- 


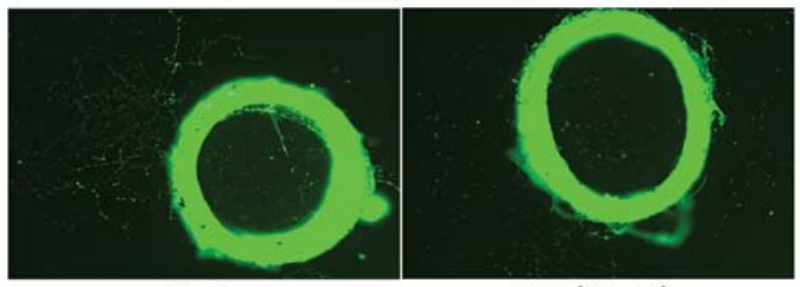

CTRL

$\operatorname{SFP}(50 \mu \mathrm{M})$

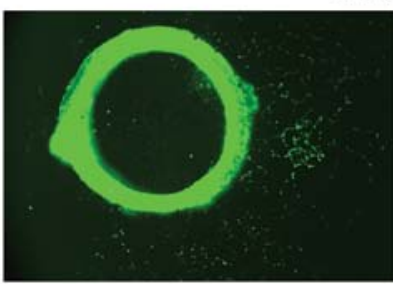

$\mathrm{MB}(10 \mu \mathrm{M})$

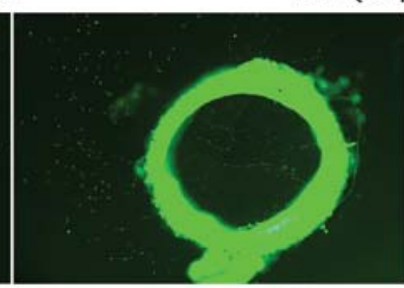

$\mathrm{MB}(20 \mu \mathrm{M})$

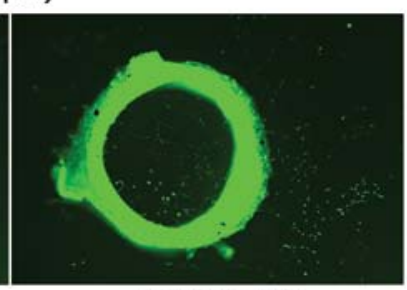

$\mathrm{MB}(40 \mu \mathrm{M})$

Figure 3. MB significantly suppresses tube formation ex vivo. MB showed an overt suppression of tube formation ex vivo evaluated by rat aortic ring angiogenesis assay. Rat aortic ring angiogenesis assay was performed as described in Materials and methods. Vehicle (DMSO) was used as a CTRL, and SFP served as the positive-CTRL. Each assay was performed in triplicate. MB, manassantin B; SFP, L-sulforaphane.

A

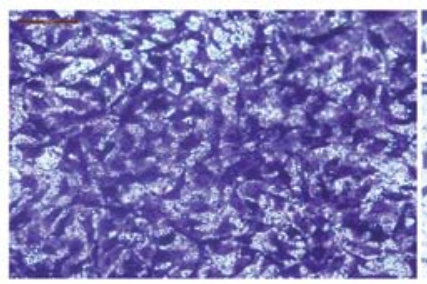

CTRL

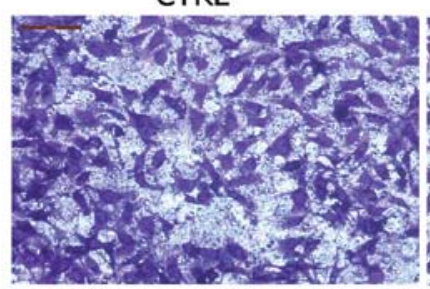

$\mathrm{MB}(10 \mu \mathrm{M})$
EA.hy 926

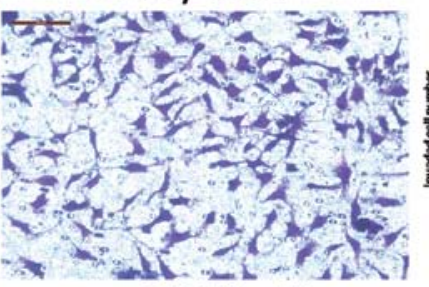

$\operatorname{SFP}(50 \mu \mathrm{M})$

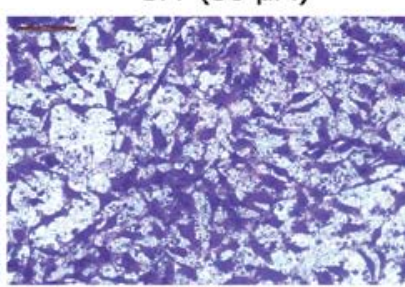

MB $(20 \mu \mathrm{M})$
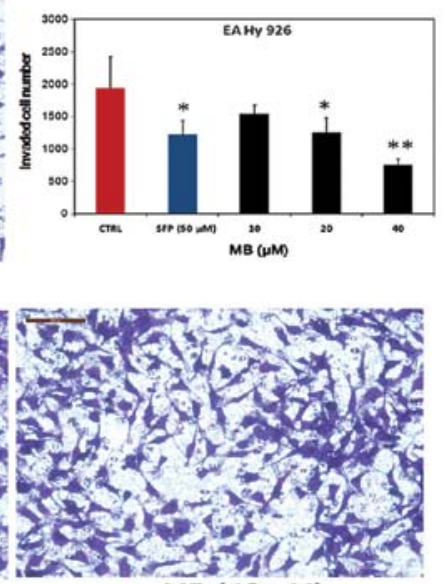

$\mathrm{MB}(40 \mu \mathrm{M})$

B

HUVECS
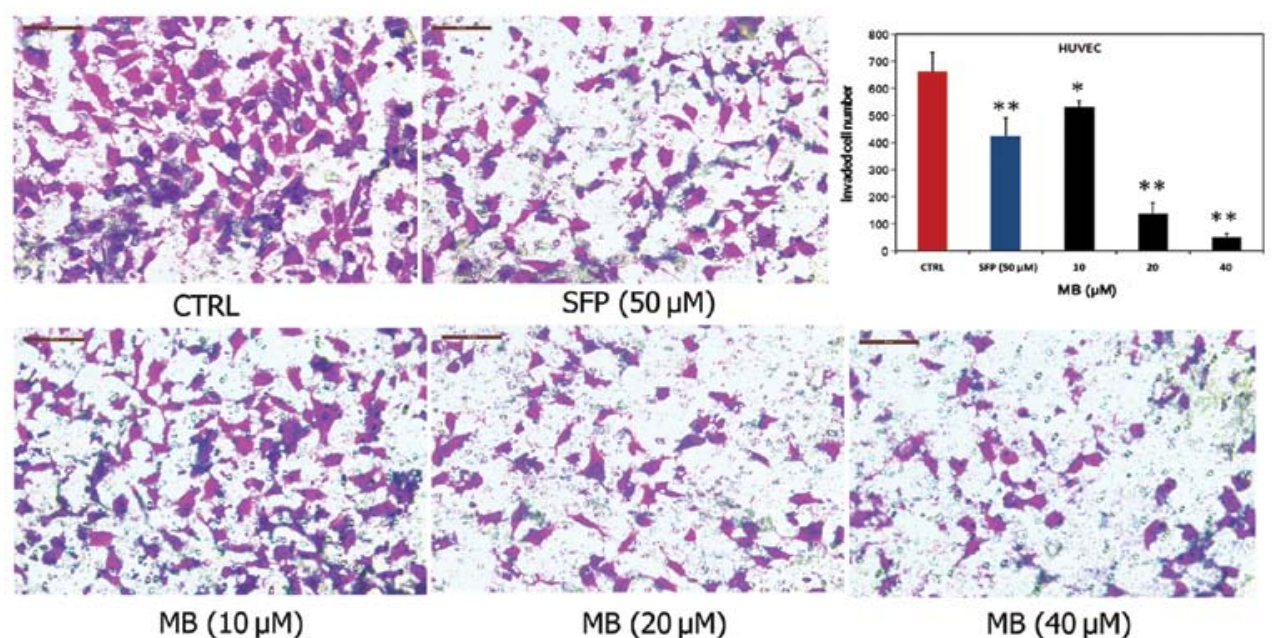

Figure 4. In vitro invasion of ECs is significantly inhibited by MB in a dose-dependent manner. (A) MB inhibited the invasion of EA.hy 926 cells in the AP48 chamber. Five fields were counted for each chamber, and each concentration was repeated in three chambers. (B) Effects of MB on invasion of HUVECs. In vitro invasion assay was carried out as described in Materials and methods. Invaded cell number was presented as means $\pm \mathrm{SD}$ percentage $(\mathrm{n}=3)$. ${ }^{*} \mathrm{P}<0.05$, ${ }^{* *} \mathrm{P}<0.01$ when compared with control (CTRL). Vehicle (DMSO) was used as a CTRL, and SFP served as the positive-CTRL. Each assay was performed in triplicate. ECs, endothelial cells; MB, manassantin B; HUVECs, human umbilical vein endothelial cells; SFP, L-sulforaphane. 
A
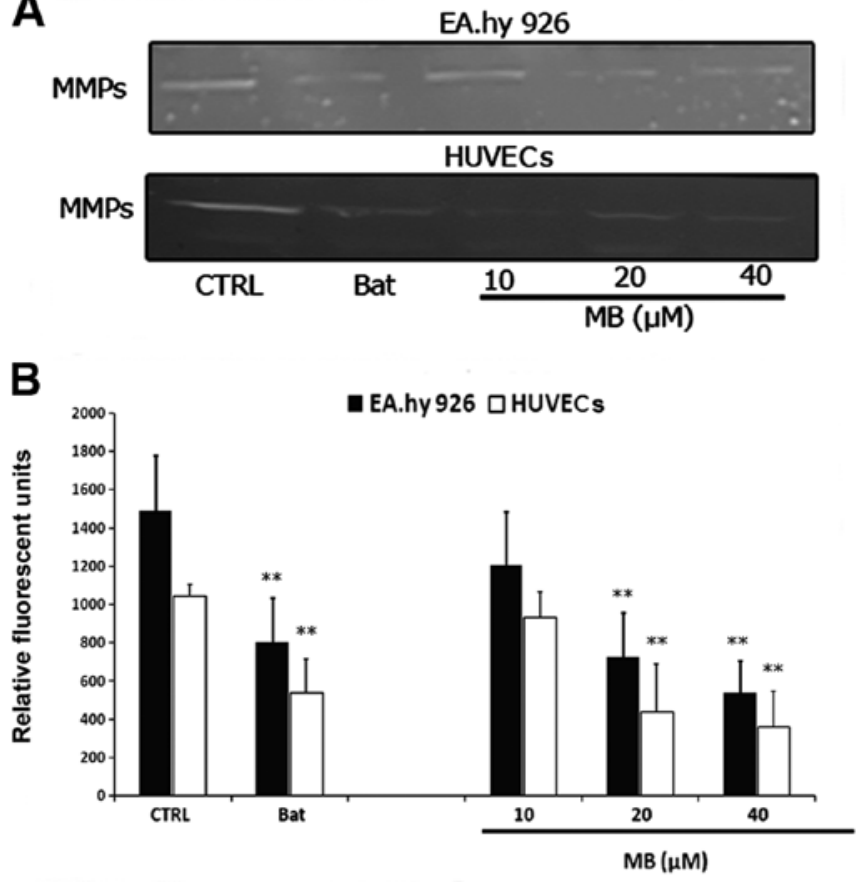

C

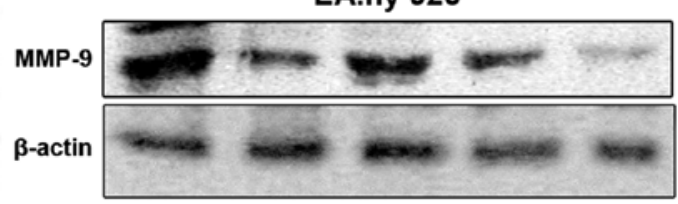

HUVECS

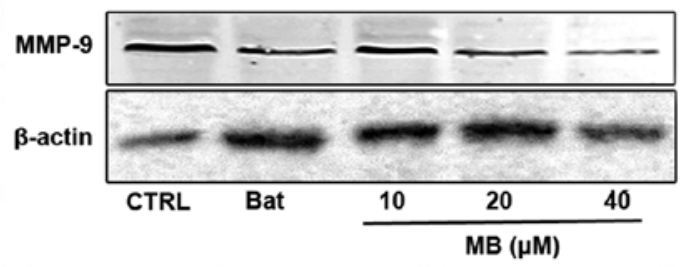

Figure 5. Effects of MB on activity of matrix metalloproteinases (MMPs) in ECs. (A) MMP protein activity was detected using gelatin zymography. (B) MMP protein activity was detected by the FRET-based MMP activity assay kit. (C) MB inhibited MMP-9 expression in ECs as detected by western blotting. Vehicle (DMSO) was used as a CTRL, and Bat served as a positive-CTRL. All treatments were conducted in the presence of $2 \mathrm{ng} / \mathrm{ml}$ VEGF. ${ }^{* *} \mathrm{P}<0.01$ compared with DMSO-treated ECs. Each assay was performed in triplicate at minimum. MB, manassantin B; ECs, endothelial cells; Bat, batimastat.

cant suppression of FRET substrate cleavage of MMPs in a dose-dependent manner (Fig. 5B). To further determine whether MB inhibited the functional activity or expression of MMPs in ECs, we analyzed the expression of MMP-9, one of the important MMPs, in EA.hy 926 and HUVECs treated with MB. As shown in Fig. 5C, MB significantly decreased MMP-9 expression in both $\mathrm{EC}$ lines in a dose-dependent manner. These data suggested that the anti-angiogenic properties of MB may be due to downregulation of expression of MMP-9 in ECs.

Effects of MB on RUNX2 activation in ECs. From current observations, we understood that Runx2 is a 'master' transcriptional factor of metastatic growth of breast cancer cells. Several genes required for the formation of metastatic foci, including mmp-9, bsp, opn and vegf, are targets of this transcriptional factor. Therefore, next we examined RUNX2 activities in ECs when cells were co-incubated with VEGF at $2 \mathrm{ng} / \mathrm{ml}$. As shown in Fig. 6A, MB had no effect on total RUNX2 expression, but caused a significant decrease of phospho-RUNX2 expression. These results indicate that MB-induced downregulation of MMP-9 expression may be associated with the suppression of RUNX2 activation.

To confirm the western blot results, Runx 2 transcription factor assay and ChIP assay were designed. First, an enzymelinked immunoabsorbent assay (ELISA)-based kit for the Runx2 transcription factor was used to analyze the effects of MB on Runx2. EC nuclear extracts incubated with MB or vehicle were prepared and the binding activity between RUNX2 with its target sequence was determined. The results showed that MB decreased the binding activity of RUNX2 to its target sequences in a dose-dependent manner (Fig. 6B). Using ChIP assay, we found that binding of RUNX2 to one of the RUNX2 binding domains (-220 bp; TGGGGTC) in the mmp-9 promoter region was inhibited by MB (Fig. 6C). Collectively, these findings suggested that the inhibitory effect of MB on MMP-9 was caused by MB inhibition of RUNX2 binding to the $m m p-9$ promoter, which may be associated with suppression of RUNX2 phosphorylation.

\section{Discussion}

MB, a neolignan isolated from the roots of $S$. chinensis, has been demonstrated to exhibit a range of activities, including anti-inflammatory (10,19-21), antiseptic (22) and antitumor activities $(11,23)$. Furthermore, MB was shown to inhibit PMA-induced ICAM-1 expression in HL-60 cells (15) and to prevent monocyte adhesion to HUVECs through the inhibition of ICAM-1, VCAM-1 and E-selectin expression stimulated by TNF- $\alpha$ (24). Due to the important roles of these adhesion molecules in tumor-induced angiogenesis, we sought to determine whether MB exerts its effects directly on ECs in angiogenesis and to identify the underlying mechanisms. In the present study, we used tube formation assay of ECs and rat aortic ring angiogenesis assay to evaluate the anti-angiogenic effects of MB. To mimic tumor angiogenesis, VEGF $(2 \mathrm{ng} / \mathrm{ml})$ was added into the culture system. Results showed that meshlike structure formation on Matrigel was significantly impaired by MB both in tube formation assay and in rat aortic ring angiogenesis assay.

Angiogenesis is the physiological process through which new capillary blood vessels grow from pre-existing vessels (1-3). However, it is also a fundamental step in the transition of tumors from a benign state to a malignant one. Tumor angiogenesis starts with cancerous tumor cells releasing signal molecules, such as VEGF or bFGF, to surrounding normal host tissues. These pro-angiogenic molecules activate ECs in the host tissues that in turn stimulate a series of steps toward the creation of new blood vessels (1-3). Breakdown of matrix by MMPs in surrounding tissues is one of the critical steps that facilitate the migration of ECs. As they migrate into the surrounding tissues, activated ECs begin to divide and organize into hollow tubes that gradually evolve into a mature network of blood vessels $(25,26)$. Based on these findings, we tested whether MB exhibits effects on the invasive and migratory behaviors of ECs. Our data showed that, in the pres- 
A

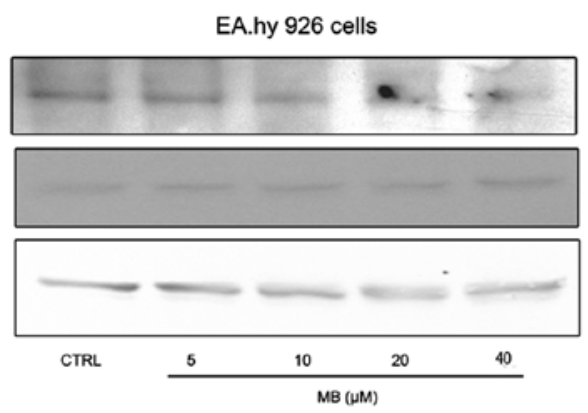

B

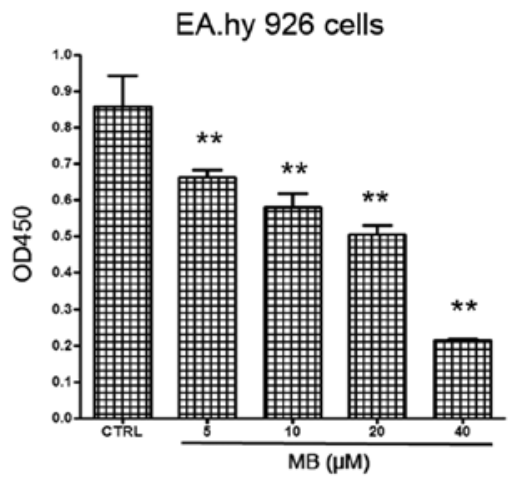

C

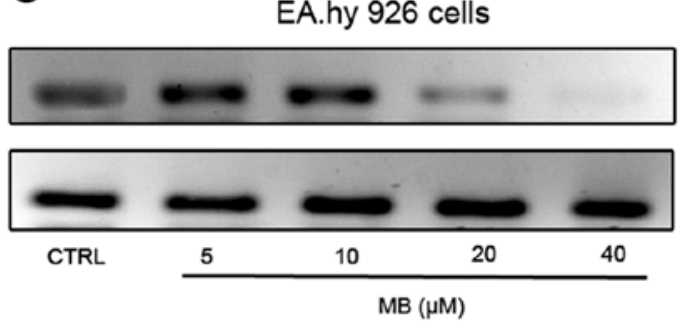

HUVECS

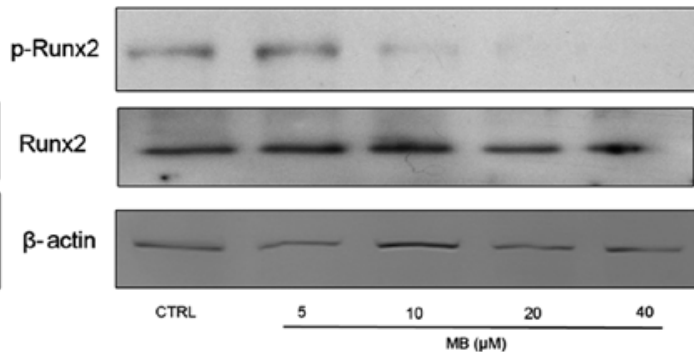

HUVECS

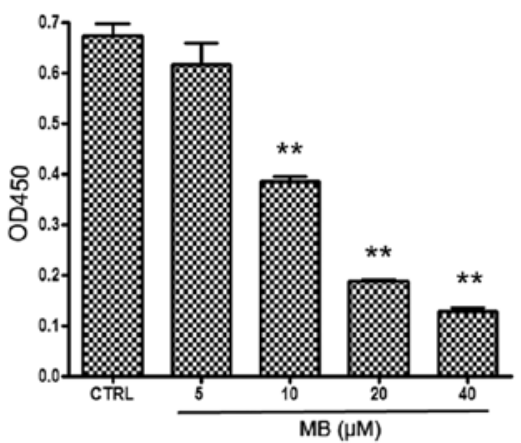

HUVECS

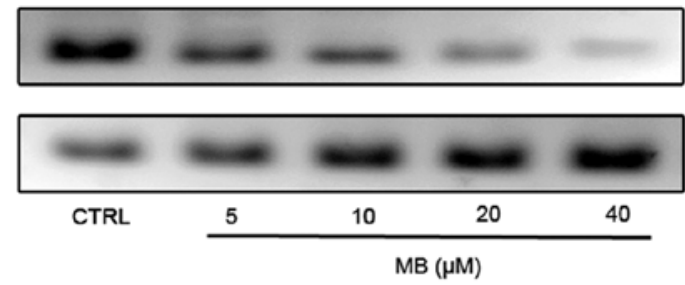

Figure 6. MB inhibits MMP-9 protein activity and expression by downregulating activation of RUNX2 transcription factor. (A) MB suppressed phosphorylation and transcription factor activity of RUNX2 in ECs. (B) RUNX2 transcription factor assay and (C) ChIP assay were used to confirm the effects of MB on RUNX2. Vehicle (DMSO) was used as a CTRL. All ECs were treated with MB for $24 \mathrm{~h}$ in presence of $2 \mathrm{ng} / \mathrm{ml}$ VEGF. "** P<0.01 compared with CTRL. MB, manassantin B; ECs, endothelial cells.

ence of $2 \mathrm{ng} / \mathrm{ml} \mathrm{VEGF}$, MB significantly inhibited invasion of HUVECs and EA.hy 926 cells through the reconstituted basement membrane.

MMPs are another major contributor to angiogenesis. These proteolytic enzymes break down matrix and allow the ECs to escape into the interstitial matrix, as seen in sprouting angiogenesis $(25,26)$. Over the past 30 years, the role of MMPs in human cancer has been widely investigated. These enzymes participate in the proteolysis of the extracellular matrix, modulation of cell adhesion, migration (27), the epithelial to mesenchymal transition (EMT) (28), processing of growth factors, and tumor-induced angiogenesis. Based on these findings, we evaluated the effects of MB on MMPs. MB treatment showed overt inhibition on MMP activities and MMP-9 expression. Collectively, these results indicate that anti-angiogenic effects of MB may be due to the suppression of expression of matrix-related proteases in ECs, resulting in inhibition of EC invasion and migration.
RUNX2, also termed PEBP2 $\alpha$ A/AML3/Cbfa1, is a critical transcription factor for osteoblastic differentiation and skeletal morphogenesis (29-31). RUNX2 belongs to the Runx family that encodes proteins homologous to Drosophila runt and has a conserved runt DNA-binding domain. RUNX2 was originally found to act as a master regulatory factor in skeletal development (32). To date, extensive evidence shows a close association between RUNX2 and cancer, and this transcriptional factor is becoming a potential target of novel anticancer agents and diagnostic approaches to cancer control $(33,34)$. RUNX2 is involved in the regulation of tumorinduced angiogenesis $(35,36)$. Sun et al (35) first reported that RUNX2 effects on EC migration and invasion may occur through the activation of protease expression, which regulates angiogenesis and tumor growth. Furthermore, Qiao et al found that RUNX2 is phosphorylated by cdc2, which may facilitate cell cycle progression and promote EC proliferation (36). 
RUNX2 is also involved in the regulation of MMP in metastatic breast cancer cells. Pratap et al investigated the role of RUNX2 in the regulation of the $m m p-9$ promoter in MDA-MB-231 and MCF-7 cells. MMP-9 was found to be a direct target of RUNX2 in metastatic breast cancer cells, and the modulation of RUNX2 activity by either forced expression or RNA interference directly affected MMP-9 expression and the invasive properties of metastatic cancer cells (37). Jiménez et al found that MMP-13, also known as collagenase-3, was highly expressed in MDA-MB-231 cells, and identified it as another target gene of RUNX2 (38). These results were also confirmed by Selvamurugan et al $(39,40)$. Thus, RUNX2 acts a 'master' transcription factor of MMP expression. Therefore, we speculated whether the inhibitory effects of MB on MMPs in ECs were associated with the downregulation of RUNX2 activity. Our western blot results demonstrated that MB significantly inhibited the levels of phospho-RUNX2, indicating that the transcriptional ability of RUNX2 on the mmp-9 promoter is impaired by MB. To confirm these findings, we used ELISA-based RUNX2 transcription factor and ChIP assays, and the results revealed that the interaction between RUNX2 and sequences in the mmp- 9 promoter was significantly inhibited by MB.

In summary, here we report that $\mathrm{MB}$, a neolignan from $S$. chinensis, inhibits the angiogenic potential induced by tumors, and the inhibitory effects are due to its ability to reduce the expression of MMP-9, a target of the RUNX2 transcription factor. These effects may be associated with inhibition of the transcriptional activity of RUNX2.

\section{Acknowledgements}

This study was supported by grants from the National Natural Science Foundation of China (grant nos. 81160530 and 81260656), the Key Research Project from the Ministry of Education of China (grant no. 211091), and the Natural Science Foundation of Jiangxi Province (grant no. 2010GQY0147).

\section{References}

1. Folkman J: Fundamental concepts of the angiogenic process Curr Mol Med 3: 643-651, 2003.

2. Adams RH and Alitalo K: Molecular regulation of angiogenesis and lymphangiogenesis. Nat Rev Mol Cell Biol 8: 464-478, 2007.

3. Carmeliet P: Angiogenesis in life, disease and medicine. Nature 438: 932-936, 2005.

4. Folkman J: Anti-angiogenesis: new concept for therapy of solid tumors. Ann Surg 175: 409-416, 1972.

5. Holleb AI and Folkman J: Tumor angiogenesis. CA Cancer J Clin 22: 226-229, 1972

6. Ferrara $\mathrm{N}$ and Kerbel RS: Angiogenesis as a therapeutic target. Nature 438: 967-974, 2005.

7. Weis SM and Cheresh DA: Tumor angiogenesis: molecular pathways and therapeutic targets. Nat Med 17: 1359-1370, 2011.

8. Bridges EM and Harris AL: The angiogenic process as a therapeutic target in cancer. Biochem Pharmacol 81: 1183-1191, 2011

9. Editorial Committee of the Administration Bureau of Traditional Chinese Medicine: Saururus Chinensis. In: Chinese Materia Medica (Zhonghua Bencao). Shanghai Science and Technology Press, Shanghai, pp2016-2017, 1998

10. Hwang BY, Lee JH, Nam JB, et al: Lignans from Saururus chinensis inhibiting the transcription factor NF- $\kappa \mathrm{B}$. Phytochemistry 64: 765-771, 2003.

11. Lee YK, Seo CS, Lee CS, et al: Inhibition of DNA topoisomerases I and II and cytotoxicity by lignans from Saururus chinensis. Arch Pharm Res 32: 1409-1415, 2009.
12. Sung SH, Kwon SH, Cho NJ and Kim YC: Hepatoprotective flavonol glycosides of Saururus chinensis herbs. Phytother Res 11: 500-503, 1997.

13. Wang EC, Shih MH, Liu MC, et al: Studies on constituents of Saururus chinensis. Heterocycles 43: 969-975, 1996.

14. Seo BR, Lee KW, Ha J, et al: Saucernetin-7 isolated from Saururus chinensis inhibits proliferation of human promyelocytic HL-60 leukemia cells via $G_{0} / G_{1}$ phase arrest and induction of differentiation. Carcinogenesis 25: 1387-1394, 2004.

15. Rho MC, Kwon OE, Kim K, et al: Inhibitory effects of manassantin A and B isolated from the roots of Saururus chinensis on PMA-induced ICAM-1 expression. Planta Med 69: 1147-1149, 2003.

16. Lu Y, Hong TG, Jin M, et al: Saucerneol G, a new lignan, from Saururus chinensis inhibits matrix metalloproteinase-9 induction via a nuclear factor $\kappa \mathrm{B}$ and mitogen activated protein kinases in lipopolysaccharide-stimulated RAW264.7 cells. Biol Pharm Bull 33: 1944-1948, 2010.

17. Watanabe K and Jaffe EA: Hypoglycemia stimulates thrombininduced PGI2 production by cultured human umbilical vein endothelial cells. Prostaglandins Leukot Essent Fatty Acids 52: 251-254, 1995.

18. Bellacen K and Lewis ECJ: Aortic ring assay. J Vis Exp 33: 1564 , 2009.

19. Lu Y, Hwang SL, Son JK and Chang HW: Manassantin B isolated from Saururus chinensis inhibits cyclooxygenase-2-dependent prostaglandin $D_{2}$ generation by blocking Fyn-mediated nuclear factor-kappaB and mitogen activated protein kinase pathways in bone marrow derived-mast cells. Biol Pharm Bull 36: 1370-1374, 2013.

20. Park HC, Bae HB, Jeong CW, et al: Effect of manassantin B, a lignan isolated from Saururus chinensis, on lipopolysaccharide-induced interleukin-1 $\beta$ in RAW 264.7 cells. Korean J Anesthesiol 62: 161-165, 2012.

21. Son KN, Song IS, Shin YH, et al: Inhibition of NF-IL6 activity by manassantin B, a dilignan isolated from Saururus chinensis, in phorbol myristate acetate-stimulated U937 promonocytic cells. Mol Cells 20: 105-111, 2005.

22. Seo CS, Lee YK, Kim YJ, et al: Protective effect of lignans against sepsis from the roots of Saururus chinensis. Biol Pharm Bull 31: 523-526, 2008.

23. Hodges TW, Hossain CF, Kim YP, et al: Molecular-targeted antitumor agents: the Saururus cernuus dineolignans manassantin B and 4-O-demethylmanassantin B are potent inhibitors of hypoxia-activated HIF-1. J Nat Prod 67: 767-771, 2004.

24. Kwon OE, Lee HS, Lee SW, et al: Manassantin A and B isolated from Saururus chinensis inhibit TNF- $\alpha$-induced cell adhesion molecule expression of human umbilical vein endothelial cells. Arch Pharm Res 28: 55-60, 2005.

25. Stetler-Stevenson WG: Matrix metalloproteinases in angiogenesis: a moving target for therapeutic intervention. J Clin Invest 103: 1237-1241, 1999.

26. Rundhaug JE: Matrix metalloproteinases and angiogenesis. J Cell Mol Med 9: 267-285, 2005.

27. Duffy MJ, Maguire TM, Hill A, et al: Metalloproteinases: role in breast carcinogenesis, invasion and metastasis. Breast Cancer Res 2: 252-257, 2000.

28. Radisky ES and Radisky DC: Matrix metalloproteinase-induced epithelial-mesenchymal transition in breast cancer. J Mammary Gland Biol Neoplasia 15: 201-212, 2010.

29. Lian JB, Stein JL, Stein GS, et al: Runx2/Cbfa1 functions: diverse regulation of gene transcription by chromatin remodeling and co-regulatory protein interactions. Connect Tissue Res 44 (Suppl 1): S141-S148, 2003.

30. Karsenty G: Role of Cbfa1 in osteoblast differentiation and function. Semin Cell Dev Biol 11: 343-346, 2000.

31. Komori T: Runx2, a multifunctional transcription factor in skeletal development. J Cell Biochem 87: 1-8, 2002.

32. Stein GS, Lian JB, van Wijnen AJ, et al: Runx2 control of organization, assembly and activity of the regulatory machinery for skeletal gene expression. Oncogene 23: 4315-4329, 2004.

33. Barnes GL, Javed A, Waller SM, et al: Osteoblast-related transcription factors Runx2 (Cbfa1/AML3) and MSX2 mediate the expression of bone sialoprotein in human metastatic breast cancer cells. Cancer Res 63: 2631-2637, 2003.

34. Shore P: A role for Runx 2 in normal mammary gland and breast cancer bone metastasis. J Cell Biochem 96: 484-489, 2005.

35. Sun L, Vitolo M and Passaniti A: Runt-related gene 2 in endothelial cells: inducible expression and specific regulation of cell migration and invasion. Cancer Res 61: 4994-5001, 2001. 
36. Qiao M, Shapiro P, Fosbrink M, et al: Cell cycle-dependent phosphorylation of the RUNX2 transcription factor by cdc 2 regulates endothelial cell proliferation. J Biol Chem 281: 7118-7128, 2006.

37. Pratap J, Javed A, Languino LR, et al: The Runx2 osteogenic transcription factor regulates matrix metalloproteinase 9 in bone metastatic cancer cells and controls cell invasion. Mol Cell Biol 25: 8581-8591, 2005.

38. Jiménez MJ, Balbín M, López JM, et al: Collagenase 3 is a target of Cbfa1, a transcription factor of the runt gene family involved in bone formation. Mol Cell Biol 19: 4431-4442, 1999.
39. Selvamurugan $\mathrm{N}$ and Partridge $\mathrm{NC}$ : Constitutive expression and regulation of collagenase- 3 in human breast cancer cells. Mol Cell Biol Res Commun 3: 218-223, 2000.

40. Selvamurugan N, Kwok S and Partridge NC: Smad3 interacts with JunB and Cbfa1/Runx 2 for transforming growth factor-beta1stimulated collagenase-3 expression in human breast cancer cells. J Biol Chem 279: 27764-27773, 2004. 